\title{
La participación política de los jóvenes en la Unión Europea
}

David Redoli Morchón*

Todos los agentes de socialización son corresponsables del éxito o fracaso del desarrollo y la integración de sus generaciones más jóvenes. Pero un peso especial recae sobre las instituciones públicas. Para llegar a ser plenos ciudadanos europeos, los jóvenes necesitan diseños institucionales que les permitan tener una presencia constante en la esfera pública de la sociedad y adquirir aceptables niveles de autonomía. Puesto que la juventud es el período por excelencia en el que se lleva adelante el proceso de producción de ciudadanos, es también responsabilidad de los organismos de gobierno de la Unión Europea, de sus Estados miembros y de sus regiones, el poner en marcha los mecanismos necesarios para facilitar la emancipación juvenil.

Los cinco grandes programas específicos para jóvenes de la Comisión Europea, Sócrates II, Juventud, Leonardo da Vinci II, Youth-Med y Tempus III, junto con el sistema europeo de información juvenil Eurodesk, deben ser aprovechados al máximo, pero hacen falta medidas de mayor alcance, como por ejemplo, la rápida adopción por parte de los gobiernos de los Estados de

* Ex-técnico del Foro Europeo de la Juventud (YFJ) para la Presidencia española de la UE 2002. Investigador de la Universidad de Salamanca. E-mail: david.redoli@map.es

V. 4

ก. 2

jul.-dez. 2004

p. $303-336$ 
la UE de las recomendaciones contenidas en el Libro Blanco de la Comisión Europea Un nuevo impulso para la juventud europea o la revitalización de los programas de juventud del Consejo de Europa.

En Europa las cohortes de menor edad van a ser protagonistas de una redefinición del modelo de sociedad que conocemos. En este sentido, el movimiento asociativo juvenil europeo, por sus dimensiones, es un actor clave a tener en cuenta. Los actuales y futuros servicios de juventud habrán de contar, en su diseño e implementación, con plataformas como el Foro Europeo de la Juventud (YFJ), una organización juvenil internacional que presta sus propios servicios al movimiento asociativo juvenil europeo al que representa y por el que trabaja.

Las estructuras del movimiento asociativo juvenil europeo enfrentan cuestiones muy similares a las que se plantean las instituciones y los responsables de las políticas públicas de juventud: ¿cómo son los jóvenes en la actualidad?, ¿cuál es el contexto sociodemográfico en el que se desenvuelven?, ¿cuáles son sus principales problemas?, ¿qué tipo de servicios demandan los jóvenes del siglo XXI? La juventud europea, asociada o no, va a enfrentar retos que ninguna de las cohortes generacionales predecesoras pudieron siquiera imaginar: la revolución tecnológica (Internet, tele-trabajos, inmediatez), la globalización (cambios fundamentales en el "multilateralismo hegemónico" de Bretton Woods y en el orden internacional de posguerra), la redefinición del Estado de bienestar (Welfare State vs Workfare State), la emergencia de los nuevos movimientos sociales (ONGs, ecologismo, pacifismo, nuevo internacionalismo, etc.) o el incremento de los flujos migratorios son sólo algunos de los desafíos más importantes que muestran las complejidades del mundo que está por llegar.

El presente trabajo justifica la importancia de potenciar los servicios de juventud prestados por la principal plataforma del movimiento asociativo juvenil europeo, el Foro Europeo de la Juventud (YFJ), como forma de robustecer la democracia participativa y el aprendizaje ciudadano entre las cohortes de menor edad.

El trabajo se encuentra estructurado en cinco secciones, articuladas de la siguiente manera: (a) La juventud europea en el siglo XXI (donde se analiza la evolución del concepto de juventud en Europa), (b) Un reto para los servicios de juventud: el retraso de la adquisición de la autonomía de los jóvenes 
en la Unión Europea (donde se analizan los factores que están condicionando la emancipación juvenil), (c) La juventud europea y el ejercicio de la ciudadanía activa (donde se examinan las consecuencias para la participación debidas a la dilatación del proceso de emancipación juvenil), (d) Las organizaciones juveniles europeas: un nuevo impulso para el desarrollo de los servicios de juventud (donde se justifican la validez y conveniencia de reforzar los servicios de juventud prestados por las asociaciones juveniles europeas) y (e) El Foro Europeo de la Juventud (YFJ) y los servicios de juventud por y para el movimiento asociativo juvenil europeo (donde se explican el funcionamiento y los principales servicios de juventud prestados por esta plataforma internacional de coordinación). Finalmente, se incluyen una sección de conclusiones y otra detallando la bibliografía consultada.

\section{La juventud europea en el siglo XXI}

Nuestras sociedades europeas están envejeciendo debido al descenso de las tasas de natalidad y al aumento de la longevidad. Baste como muestra el siguiente dato: entre 2000 y 2020 , el porcentaje de personas entre 65 y 90 años de edad pasará de representar el 16\% de la población total de la Unión Europea a significar el 21\%, mientras que las personas de la cohorte de edad correspondiente al intervalo 15-24 años solamente representarán el $11 \%$ de la población de la UE (en comparación con el 12,4\% que hoy significan) (Comisión Europea, 2002).

De no invertirse esta tendencia, será un elemento crucial de las políticas públicas futuras el reorganizar el relevo generacional, a fin de evitar la ruptura de los sistemas sociales actuales y, muy especialmente, de los sistemas de pensiones, del sistema educativo y del mercado laboral.

Todas las sociedades se reproducen a sí mismas a través de la transmisión de valores, de creencias y de instituciones de unas generaciones a otras, pero se mejoran con las nuevas ideas, los nuevos valores, las nuevas creencias y las nuevas instituciones aportadas por las generaciones más jóvenes. La juventud es, por tanto, un actor fundamental a tener en cuenta en el proceso de construcción y reproducción de cualquier sociedad (Redoli, 2002, p. 32). 
La juventud suele definirse como el periodo en el que se pasa de la vida dependiente propia de la niñez a las formas de independencia propias de los adultos. En otras palabras: la juventud es la fase biológica de transición entre la infancia (heteronomía) y la madurez (autonomía), transición durante la que se inicia la proyección social independiente de la persona en el contexto de su comunidad.

Nunca se había hablado tanto ni con tanta intensidad de los jóvenes como se hace en la actualidad una renovada influencia en el campo político y constituyen un grupo consumidor y productor relevante. Tal es la importancia que cobra la juventud en nuestros días, que la Convención Europea ${ }^{1}$ decidió recientemente organizar la Convención Joven para consultar con los jóvenes del continente las futuras reformas que enfrentará la Unión Europea. $^{2}$

No obstante, "ser joven" no tiene una definición muy clara, puesto que es una construcción: ha aumentado la visibilidad de la juventud, los jóvenes tienen artificial que tiende a tener significados diferentes según el contexto histórico, social, legal, político y cultural del que se trate. Luis Enrique Alonso lo describe de esta manera:

La juventud es, además de un periodo de edad física determinada que se experimenta personalmente y se percibe intuitivamente, una construcción social que viene determinada por el conjunto de representaciones, convenciones, instituciones y justificaciones que la articulan y regulan dentro de la vida colectiva de las diferentes sociedades. Por tanto, gran parte de las formas y modos de vida de los jóvenes dependen de cómo se construye socioculturalmente el lugar que los diferentes colectivos juveniles ocupan en la sociedad y de cómo las instituciones, tanto informales (costumbres, tradiciones, tópicos, valores, discursos, etc.) como formales (normas jurídicas, administrativas y organizativas de todo tipo, y a todos los niveles políticos), generan el espacio en el que se desenvuelve su integración en los procesos generales de producción y reproducción económica y social. (Alonso, 2000, p. 43).

1 La Convención Europea es una institución creada ad hoc para sentar las bases de la refundación de la Unión Europea tras la ampliación a 25 Estados miembros que tendrá lugar a partir de 2005.

2 La primera reunión de la Convención Joven se celebró en Bruselas (Bélgica) entre el 9 y el 12 de julio de 2002. Todos los documentos producidos por la Convención Joven pueden consultarse en la página web del Foro Europeo de la Juventud <www.youthforum.org $>$. 
Lo que hoy entendemos por "juventud" es un concepto relativamente nuevo, resultado de una serie de acontecimientos históricos habidos en occidente durante los últimos 300 años (surgimiento de los Estados-nación, Revoluciones americana, inglesa y francesa, Revolución Industrial, guerras mundiales, posmodernidad...). Como fruto de nuestras circunstancias históricas, se ha institucionalizado un orden social que tiene muy en cuenta la estructura de edades de sus ciudadanos, a los que se les atribuyen expectativas, pautas de comportamiento, conductas sociales e, incluso, legislaciones específicas, en función de la franja de edad en la que se encuentren ubicados.

En el continente europeo no siempre ha existido la misma idea de lo que significa ser joven, ni se ha correspondido con las mismas edades. Así, durante la Edad Media y el Antiguo Régimen, la juventud se definía como una condición de género masculino que denotaba la pertenencia a los estamentos más altos de la sociedad. La juventud era el periodo de tiempo durante el cual se formaba a los futuros nobles y aristócratas para reproducir las costumbres de la posición social adscrita a su linaje. Más tarde, iniciada ya la Ilustración, la juventud se convirtió en la categoría de edad en la que la educación debía intervenir muy intensamente para entrenar las virtudes cívicas y potenciar el desarrollo de las capacidades del joven (Giner et al., 1998, p. 413).

A principios del siglo XIX la juventud, se describía como un colectivo generacional inconformista y abanderado de la razón por encima de los sentimientos. Décadas después, en el periodo prefordista, sólo se podría hablar de juventud asociada a la burguesía, puesto que en las clases obreras la adolescencia daba paso inmediato a la vida laboral, sin periodo transicional de ningún tipo. Entrado el siglo XX, la juventud, producto de la sociedad de consumo de masas en los países industrializados, pasó a ser el motor de cambios históricamente muy significativos y se diversificó a través de subculturas juveniles con sistemas de valores y elementos simbólicos propios. Tras la Segunda Guerra Mundial, y en el contexto de la era del fordismo, del Estado de bienestar y del pacto keynesiano, la juventud, asignada a un tramo de edad convencionalmente ubicado entre los 15 y los 25 años, se convirtió en un tipo ideal de transición de la adolescencia al mundo del trabajo y, por ende, a la vida adulta (Alonso,2000, p. 27-28). 
En los albores del siglo XXI, marcados por el proceso de la globalización económica e iniciado un orden social posmoderno que comienza a conocerse como "la sociedad del riesgo", (Beck, 1998) "la sociedad individualizada" (Bauman, 2001,) o "la era de la información" (Castells, 2000), se aprecian cambios decisivos de pautas y roles que afectan a la estructura de edades en occidente. Las rápidas transformaciones políticas, la mundialización de unos procesos económicos que parecen lejanos y distantes, los vertiginosos cambios culturales, la complejidad y la flexibilidad de un mercado laboral cada vez más exigente, el surgimiento de nuevas estructuras sociales altamente complejas, la constante redefinición de las identidades personales y colectivas y la difuminación de las instituciones tradicionales son sólo algunos de los novedosos procesos a los que se enfrentan diariamente tanto jóvenes como adultos (Beck, 2000, p. 9-23).

Como consecuencia de estas condiciones sociales, la socialización ${ }^{3}$ de la juventud ha entrado en un profundo periodo de transformación. Los jóvenes del siglo XXI muestran elementos idiosincrásicos que les diferencian de generaciones inmediatamente anteriores: hoy acceden al empleo y fundan una familia más tarde de lo que era habitual, intercalan periodos de trabajo y de estudio y, sobre todo, sus itinerarios personales son mucho más intermitentes, variados e impredecibles que en el pasado. La escuela o la universidad, el empleo y el entorno social ya no desempeñan la misma función integradora de antaño y, además, se independizan a edades muy tardias. (Comisión Europea 2002, p. 6). Con estas condiciones, comienza a apreciarse una "juvenilización de la sociedad", en la que un colectivo con edades cada vez más avanzadas (y de límites cronológicos borrosos) sigue adoptando pautas de comportamiento y conductas hasta hace pocos años consideradas exclusivas de las cohortes de menor edad (Giner, 1998, p. 413).

3 La socialización es aquel proceso mediante el que aprenden y se forman las personas para desenvolverse en su sociedad. Los agentes de la socialización son las personas y las instituciones con las que el individuo se relaciona y de las que aprende normas de conducta y de valores. El proceso de socialización dura toda la vida, aunque se suelen distinguir la socialización primaria (correspondiente a la infancia, que es cuando se interiorizan los elementos más importantes para la vida en sociedad, como el lenguaje), la socialización secundaria (correspondiente a la adolescencia y la madurez, que es cuando se interiorizan los valores más abstractos, como la ideología política) y la socialización terciaria (correspondiente a la vida adulta, que es cuando los individuos relativizan lo aprendido anteriormente e incorporan nuevos valores). 
Como algunos autores llegan a afirmar, "en nuestras sociedades está aumentando la incongruencia entre la maduración biológica y relacional de la juventud y los ritmos de paso hacia la sociedad adulta. Al tiempo que los desarrollos biosociales son más precoces, los procesos de emancipación se concluyen más tardíamente" (Martín Serrano, 2002, p. 104). Las nuevas situaciones de riesgo para la vida y de competitividad internacional (reflejadas a todos los niveles), contribuyen a que hoy ya no resulte extraño catalogar como jóvenes (ateniéndonos a la definición aquí proporcionada) a personas de hasta 36 años de edad (edad que roza el ecuador de la existencia de una persona en una sociedad donde la expectativa de vida ronda los 75-80 años). ${ }^{4}$

La "juvenilización de la sociedad" puede entenderse, por tanto, como una consecuencia del nuevo modo de regulación posfordista o posmoderno, puesto que los procesos de transición al mundo del trabajo están tan marcados por la flexibilidad, la volubilidad y la imprevisibilidad que los jóvenes (y los no tan jóvenes) no tienen más alternativa que prolongar la etapa juvenil para seguir esforzándose en culminar satisfactoriamente su proceso de emancipación en un entorno tremendamente desarticulado. ${ }^{5}$ En este sentido, el grueso de las políticas públicas y los servicios de juventud que desarrollan las instituciones regionales, estatales y europeas en los albores del siglo XXI empiezan a estar muy orientados hacia la resolución de la problemática de la inserción laboral de los jóvenes y la reducción y suavización del complejo y alargado proceso de emancipación juvenil. ${ }^{6}$

El periodo de juventud resulta hoy extremadamente largo. De esta manera, "ser joven" está comenzando a dejar de ser un estado de transición relacionado con unas determinadas edades (como había venido siendo desde mitad del siglo XX), para convertirse en un estado, en un "estar en la juventud", estado caracterizado ya no por la edad, sino por experimentar una situación de inestabilidad y de dependencia y por las dificultades existentes para

4 Por primera vez en nuestras sociedades, la juventud dura ya más tiempo que la infancia.

5 Caracterizado, como ya ha sido mencionado, por la adopción de la flexibilidad, la competitividad, la individualización, la desregulación y la privatización de lo público como principios rectores del ordenamiento social y económico.

6 McNeish, Wallace; L'Oncle, Patricia. Documento de trabajo presentado en la Conferencia europea para investigadores y técnicos "Jóvenes y políticas de transición en Europa". Madrid, 6-8 de junio de 2002 . 
emanciparse plenamente y llegar a la "edad de adulto autónomo". ${ }^{7}$ Llegar a la fase en la que debería obtenerse la independencia personal y producirse la autonomía del individuo a través de la adquisición de responsabilidades, derechos y deberes frente a la sociedad, se ha convertido en una ingente tarea (Martín Serrano, 2002, p. 104).

Esta situación contribuye a que la juventud se constituya hoy en día como un conjunto de personas extremadamente heterogéneo, mostrando unas diversidades sociales, económicas y culturales (tanto intra como intergrupales) que complejizan la aplicación de políticas públicas que ayuden a resolver, de modo global, el problema de la "juvenilización de la sociedad" y evitar que "ser joven" se convierta en sinónimo de existencia en precario.

Con estos preocupantes parámetros, el proceso de emancipación de los jóvenes se está convirtiendo en objeto central de estudio y análisis en todos los países de occidente y, muy especialmente, en la Unión Europea (IBRD, 2001).

Tal y como refleja el informe The Social Situation in the European Union 2001, la juventud del continente está dilatando su proceso de emancipación debido, principalmente, a la compleja situación del mercado laboral. Las cohortes de menor edad enfrentan un difícil acceso al mercado de trabajo, viendo limitada su plena integración en la sociedad como ciudadanos europeos independientes. La tasa media de desempleo juvenil en la Unión Europea ${ }^{8}$ roza en la actualidad el $18 \%$, aunque existen significativas variaciones entre el 5\% de la tasa de desempleo juvenil de Luxemburgo, Austria y Holanda y el $30 \%$ exhibido por Grecia, España e Itália (Eurostat, 2002, p. 86). A estas tasas de desempleo hay que sumarle el impacto de la precariedad del mercado laboral. En este sentido, España resulta especialmente perjudicada puesto que los índices de trabajo precario son tres veces superiores a la media europea y en las franjas juveniles este índice se dispara y llega a situarse hasta seis veces por encima de la media de la Unión (Serrano, 1999, p. 196).

Esta situación de precariedad que sufren los europeos mas jóvenes, contrasta con sus altos niveles educativos y, por ende, con sus potenciales pro-

7 Entendiendo por 'autonomía' la ocupación de un puesto en la sociedad, que proporcione los recursos necesarios para poder emanciparse económica y residencialmente de la familia de origen.

8 Para el intervalo de 15 a 24 años. 
ductivos. Baste un dato como muestra: en 1999, el 74\% de los jóvenes de la UE entre 25 y 29 años de edad habían completado la educación secundaria, cuando sólo el 49\% de los que hoy tienen entre 50 y 64 años lo han hecho (Eurostat, 2002, p. 76).

Tal es la importancia que está cobrando la actual situación social de los jóvenes en los 15 Estados miembros, que la Comisión Europea decidió en 1999 redactar un Libro Blanco sobre este tema. ${ }^{9}$ Tras llevar a cabo un proceso de consulta desde mayo de 2000 hasta marzo de 2001, ${ }^{10}$ la Dirección General de Educación y Cultura de la Comisión Europea presentó en Gante (Bélgica), en noviembre de 2001, el Libro Blanco Un nuevo impulso para la juventud europea, documento que reconoce la necesidad de encontrar nuevos marcos de cooperación en el ámbito de la juventud y de satisfacer las demandas de un colectivo muy vulnerable y hoy necesitado de un especial apoyo institucional a todos los niveles (local, regional, estatal y supranacional).

De acuerdo a este Libro Blanco, la autonomía y la plena emancipación se han convertido en una de las principales reivindicaciones de los jóvenes. En estos términos se expresa la propia Comisión Europea a este respecto:

La autonomía se basa en los medios que se les conceden [a los jóvenes], y, en especial, los medios materiales. A este respecto, es de capital importancia la cuestión de los ingresos. La juventud se ve afectada por las políticas de empleo, protección social y ayuda a la inserción, así como por las políticas de vivienda y transporte. Estas políticas son necesarias para permitir que los jóvenes consigan mas rápidamente la autonomía, y deberían desarrollarse teniendo en cuenta sus puntos de vista y sus intereses, así como aprovechando los logros y las experiencias propias de las políticas de la juventud. Los jóvenes rechazan que las políticas de la juventud se circunscriban a ámbitos específicos, ya que quieren ser una parte activa de la sociedad y se sienten afectados por las políticas relacionadas con las diferentes facetas de sus condiciones de vida (Comisión Europea, 2002, p. 19).

9 Los Libros Blancos publicados por la Comisión Europea son documentos que contienen propuestas de acción comunitaria en un sector específico. También se definen como un conjunto oficial de propuestas para un ámbito político concreto. El Libro Blanco "Un nuevo impulso para la juventud europea", es, por tanto, un trabajo que puede servir como base para la elaboración de una legislación comunitaria específica en materia de juventud.

10 Proceso de consulta que tuvo como destinatarios a jóvenes europeos de todos los orígenes, así como a las organizaciones juveniles, a la comunidad científica, a los responsables políticos $\mathrm{y}$ a sus administraciones. 
Es, por tanto, reconocido el hecho de que los jóvenes experimentan un profundo proceso de transformación, que se traduce en la "prolongación de la juventud." Asimismo, a tenor de los datos suministrados por las instituciones locales, regionales, nacionales y europeas, se constata oficialmente la desaparición de los modelos colectivos tradicionales de integración social en favor de trayectorias personales cada vez más individualizadas que necesitan de nuevas fórmulas de compromisos. La siguiente tabla, construida tomando datos suministrados por el estudio Eurobarometer 2001 Survey on Youth (Eurobarometer 55.1) de Eurostat y la Dirección General de Educación y Cultura de la Comisión Europea y el informe The Social Situation in the European Union 2001 de Eurostat y la Dirección General de Empleo y Asuntos Sociales de la Comisión Europea, ofrece una "foto fija" del estado de la juventud en la Unión Europea:

\section{Tabla 1}

Cifras definitorias de la juventud europea (año 2001)

\begin{tabular}{|c|c|c|}
\hline TEMA & \multicolumn{2}{|l|}{ DATOS } \\
\hline Número total de efectivos jóvenes & \multicolumn{2}{|c|}{$\begin{array}{c}\text { 76.438.200 de jóvenes } \\
\text { (15-29 años) }\end{array}$} \\
\hline Porcentaje de jóvenes en el Parlamento Europeo & $4 \%$ & (menores de 35 años) $)^{11}$ \\
\hline Tasa de asociacionismo juvenil & $50 \%$ & (15-24 años) \\
\hline $\begin{array}{l}\text { Peso porcentual de la población juvenil } \\
\text { sobre el total de la población de la UE }\end{array}$ & \multicolumn{2}{|c|}{$20,4 \%$ (15-29 años) } \\
\hline $\begin{array}{l}\text { Porcentaje de jóvenes de la UE con educación } \\
\text { secundaria completada }\end{array}$ & $74 \%$ & (25-29 años) \\
\hline $\begin{array}{l}\text { Porcentaje de jóvenes de la UE con educación } \\
\text { universitaria completada }\end{array}$ & $20 \%$ & (mayores de 25 años) \\
\hline Media porcentual del paro juvenil en la UE & $18 \%$ & (15-24 años) \\
\hline Edad media de acceso a la maternidad en la UE & & 29 años \\
\hline $\begin{array}{l}\text { Porcentaje de jóvenes que se declaran } \\
\text { plenamente emancipados en la UE }\end{array}$ & $35 \%$ & (15-24 años) \\
\hline
\end{tabular}

11 Martínez, 2000, p. 272. 


\section{Un reto para los servicios de juventud: el retraso de la adquisición de la autonomía de los jóvenes en la unión europea}

La emancipación juvenil (o la adquisición de la plena autonomía) es reconocida como uno de los principales problemas que enfrentan los jóvenes de la UE, de ahí que tanto el movimiento asociativo juvenil europeo como las instituciones públicas estén, cada vez más, prestando servicios orientados a facilitar los procesos conducentes a la adquisición de la autonomía.

La emancipación es el proceso clave mediante el cual los jóvenes se integran en el mundo adulto y adquieren lo que la mayor parte de los sociólogos de la juventud definen como el "nacimiento social autónomo del individuo". Los estudios existentes sobre emancipación juvenil aceptan la existencia de cuatro componentes esenciales que definen el grado y la calidad de la inserción en la vida adulta y la autonomía de los jóvenes (Garrido, 1996):

\section{- La formación o cualificación}

Constituye el caudal de conocimientos y habilidades técnicas (capital humano) adquiridas por el joven para desempeñarse activa, productiva y establemente en el mercado laboral. El acceso al trabajo (y al tipo de trabajo) estará, en gran medida, definido por la titulación adquirida, así como el lugar social ocupado vendrá determinado por el reconocimiento público de la cualificación profesional como herramienta productiva.

\section{- El empleo}

Este es el segundo de los elementos que caracterizan el proceso de emancipación de los jóvenes. El acceso a un lugar productivo en el que se obtenga una remuneración económica (capital financiero) significa la obtención de la independencia económica y, consecuentemente, la autonomía financiera. La importancia del trabajo en el proceso de emancipación es crucial a la hora de enfrentar su transición a la vida adulta.

\section{- El domicilio autónomo}

Dadas las actuales y aún vigentes estructuras familiares predominantes en el sur de Europa, la residencia propia (capital físico) se constituye en un elemento crucial en el proceso emancipador al ser el principal territorio físico donde el joven puede ejercer un amplio margen de libertad libre del control social familiar. Es, además, el lugar de referencia (domicilio) en el que la persona es localizable para las instituciones y para el resto de las relaciones personales. 


\section{- El ambiente relacional}

El cuarto de los recursos cuya adquisición permite la emancipación juvenil es el ambiente relacional mínimo que sustituya al que provee la familia de origen ( $\mathrm{ca}$ pital social). El ambiente relacional permite satisfacer las necesidades afectivas y sexuales básicas y está configurado por los amigos, los colegas profesionales, los compañeros de trabajo, los parientes y, principalmente, por la pareja. El proceso de formación de la pareja (en sus múltiples variantes) es el elemento básico del proceso adquisitivo de la integración juvenil en el mundo adulto, asegurando el proceso de la reproducción social.

Teniendo en cuenta estos cuatro factores, el proceso de emancipación juvenil puede abordarse desde un punto de vista procesual: la transición que lleva a los jóvenes a la vida adulta es una trayectoria en la que se van logrando diferentes adquisiciones, las cuales van definiendo posiciones sociales distintivas. No obstante, tal y como se ha expuesto en el apartado anterior, estas adquisiciones tienen hoy en día ritmos y lógicas muy complejas, especialmente en el contexto de las sociedades industrializadas contemporáneas (Roche, 1999). Cuestiones como el desempleo, la inestabilidad en el puesto de trabajo, los bajos salarios, las dificultades para la iniciativa empresarial o el encarecimiento del precio de la vivienda (Equipo de Investigación de Provivienda, 2001) aparecen como importantes elementos limitadores de la autonomía de los más jóvenes y provocan que hoy se produzca un considerable retraso en el proceso de emancipación juvenil (viéndose tremendamente afectados los canales de acceso a los capitales físicos y financieros, y deteriorados, como efecto colateral, los capitales sociales y humanos).

En este sentido, la juventud de la Unión Europea está enfrentando trayectorias muy irregulares a la hora de emanciparse. El profesor Lorenzo Cachón las sintetiza de la siguiente manera, agrupándolas en seis tipos de trayectorias hacia la autonomia (Cachón, 2002):

\section{1) Trayectorias de éxito precoz}

Desarrolladas por jóvenes con altas expectativas de carrera profesional. Suelen corresponderse con jóvenes que han realizado una carrera universitaria excepcional y que han conseguido establecerse profesionalmente en sus tres primeros años de vida laboral. 


\section{2) Trayectorias obreras}

Configuradas por jóvenes con escasos niveles formativos y orientados hacia el trabajo manual o poco cualificado. Estas trayectorias son particularmente vulnerables a los cambios del mercado de trabajo. Más que tratarse de opciones profesionales personales son alternativas laborales condicionadas por las ofertas de empleo disponibles en sectores como la construcción o la agricultura.

\section{3) Trayectorias de adscripción familiar}

Basadas en el acceso a puestos de trabajo en empresas, negocios o explotaciones familiares.

\section{4) Trayectorias de aproximación sucesiva}

Definidas por altas expectativas de mejora social y profesional en un contexto altamente indefinido. Es una modalidad de transición dominada por el tanteo, lo que retrasa la emancipación familiar ya que presupone una educación prolongada, el atravesar múltiples experiencias laborales (en ocasiones en situación de precariedad o subocupación) y la consecución de logros parciales. Es una trayectoria de inserción dominada por el ajuste continuo de expectativas, normalmente a la baja.

\section{5) Trayectorias de precariedad}

Definidas por tres características básicas: experimentación de situaciones intermitentes de paro, fuerte rotación laboral y subocupación. Se trata de unos itinerarios que implican resultados escasamente constructivos en el mercado de trabajo y que desdibujan la trayectoria profesional del joven que las padece.

\section{6) Trayectorias de desestructuración}

Desarrolladas en la economía marginal o en formas de economía sumergida. Las expectativas de partida son bajas y la formación reglada es limitada. Este tipo de trayectorias se caracteriza por presentar situaciones de paro crónico y entradas esporádicas en el mercado de trabajo secundario, de forma errática y sin posibilidad de acceder a la plena inserción laboral.

En los últimos 20 años las trayectorias profesionales predominantes entre los jóvenes europeos nacidos entre 1970 y 1984 parecen ser las tres últimas, al contrario de lo que sucedió desde los años 50 hasta mediados de los 70, donde los dos primeros tipos de trayectorias fueron los más típicos (IRIS, 2002). 
Desde el final de la II Guerra Mundial (1945) hasta la década de los 70, en el contexto del mundo occidental desarrollado, el abandono de una posición socialmente subordinada y dependiente y la adquisición de otra posición de independencia sucedía de forma relativamente pautada y predecible. En la actualidad, la transición a la vida adulta ya no es lineal, sino que el proceso está jalonado de interrupciones, de entradas y de salidas de los diversos sistemas socializadores, del mercado laboral, del sistema educativo. Ya no existen las transiciones lineales y previsibles de antaño, y, en cambio, los jóvenes atraviesan varias y diversas transiciones. Ahora el paso a la vida adulta está desestandarizado, es más complejo, cambiante y, en ocasiones, contradictorio. El Investigador Principal del Instituto de Ciencias Sociales de la Universidad de Lisboa, José Machado Pais, lo explica de esta manera:

En las décadas que siguieron a la posguerra, las transiciones de los jóvenes se asemejaban a viajes en ferrocarril. Los jóvenes, dependiendo de la clase social, el género o las cualificaciones académicas, se embarcaban en diferentes trenes con destinos predeterminados. Las oportunidades para cambiar de destino o de trayecto eran limitadas. En claro contraste, durante las dos últimas décadas, las transiciones de los jóvenes podrían compararse con viajes en automóvil. El conductor del coche se encuentra en condiciones de seleccionar el itinerario del viaje entre un vasto numero de alternativas... Las decisiones del conductor ya no garantizan un viaje con destino cierto o por rutas predeterminadas. $\mathrm{Y}$ esto, porque el terreno en el que se dan las transiciones presenta caracteres cada vez más laberínticos. En el laberinto de la vida, como en el de la circulación automovilística, surgen con frecuencia sentidos obligatorios y prohibidos, desvíos, caminos que parecen haberse ya cruzado, o por los que se ha pasado varias veces: es ese retomar los caminos lo que provoca una sensación de desorientación, de confusión, de perdición (Machado Pais, 2002, p. 89).

Es mayoritario el diagnóstico que asegura que los procesos de emancipación juvenil y de integración social se desdibujan y que las instituciones socializadoras tradicionales (familia, mercado, escuela, sistema laboral,...) sufren profundas transformaciones en un escenario donde los jóvenes tienen un elevado riesgo de perderse y de entrar en lo que en el mundo académico se conoce como "trayectorias fallidas" (Morch et al, 2002, p. 31). En la Conferencia europea para investigadores y técnicos "Jóvenes y políticas de transición en Europa ${ }^{, 12}$ se apuntaron, entre otras, cinco tesis que, en lo referido al proceso de

12 Organizada por el Instituto de la Juventud de España (INJUVE), la Asociación Regional y Europea de Análisis (AREA) y el Instituto de Innovación Regional y Ciencia Social de Alemania (IRIS). Madrid, 6-8 de junio de 2002. 
socialización que experimentan los jóvenes en la actualidad, empiezan a ser confirmadas por múltiples estudios e investigaciones. Estas tesis son las siguientes:

1) Los modelos de socialización que sirvieron para las generaciones juveniles educadas en las sociedades industriales estaban orientados a lograr la más rápida incorporación al sistema productivo y reproductivo de la juventud. Este diseño de socialización juvenil ha entrado en crisis para dejar paso a un modelo mucho más individualizado y fragmentado que rompe con la continuidad entre la etapa de formación y la de plena inserción laboral.

2) El nuevo modelo de socialización de la juventud está tratando de realizar, al mismo tiempo, dos funciones difícilmente complementarias: a) preparar a los jóvenes para que logren "salir de la juventud", pero a un plazo mucho más largo que antaño, y b) socializar a los jóvenes para que, dadas las dificultades existentes para que se produzca la plena emancipación, acepten "permanecer en la juventud" de forma indefinida.

3) El funcionamiento de las nuevas formas de socialización está promoviendo el regreso a un sistema familiar de estratificación social, donde la familia de procedencia influye sobremanera en la condición social futura de las personas jóvenes. El peso principal del cambio social vuelve a transferirse a las familias, que determinan cada vez más la reproducción de las clases y de las posiciones sociales. El sistema educativo está perdiendo progresivamente capacidad para promover la igualdad de oportunidades y en el sistema laboral también se van limitando las oportunidades de ascenso social.

4) El nuevo modelo de socialización de la juventud no funciona congruentemente. No se dan aún ni las condiciones materiales ni el reconocimiento colectivo que son necesarios para producir un ajuste entre el ser joven y lo que se pide social y económicamente para llegar a ser adulto.

5) Las contradicciones que tienen las nuevas e indefinidas pautas de socialización generan entre los jóvenes sentimientos de ansiedad. Esta situación fomenta comportamientos anómicos ante la evidencia de enfrentar un futuro tremendamente incierto, dando lugar a que crezca el número de jóvenes inseguros (Martín Serrano, 2002).

En nuestra sociedad contemporánea el proceso de emancipación a la vida adulta es, por tanto, reversible e intermitente, representado en lo que muchos investigadores han categorizado como "la socialización en zigzag" 
o "la yo-yoización de la juventud" (haciendo alusión al juguete que, a golpes, va y viene enroscándose sobre sí mismo en una cuerda). La metáfora de las "transiciones yo-yo" explica el principio de incertidumbre que domina el futuro de la juventud en el contexto de la globalización, futuro determinado por un presente en el que el joven vive como adulto y deja de hacerlo, entra y sale del mercado laboral y del sistema educativo, se pasa de la dependencia a la autonomía y vice versa (Machado Pais, 2002, p. 99). Hoy los opuestos estudiante/no estudiante, trabajador/parado, soltero/casado están superados por una multiplicidad de nuevos estatus intermedios y reversibles, más o menos transitorios o precarios que convierten la etapa juvenil en un auténtico "dilema del laberinto", cuyo precio es la falta de proyectos concretos de vida (Hernández Aristu, 2002, p. 126).

Las consecuencias personales de la excesiva prolongación de la situación en la que se encuentra el proceso de emancipación juvenil pueden ser devastadoras, puesto que erosionan la identidad social y la autoestima de la mayoría de los jóvenes que las padecen (Sennett, 2000) y dificultan el ejercicio de la ciudadanía activa al socializar personas desorientadas, inseguras y determinadas por las "biografías del riesgo" (Giddens, 1996). La “yo-yoización de la juventud" y la "juvenilización de la sociedad" tienen una alta correspondencia con un nuevo orden social que rompe con el pacto intergeneracional de posguerra e instaura un modelo que flexibiliza, desregula, privatiza y reduce el peso del Estado. El adelgazamiento del Estado provoca que las funciones redistributivas de las administraciones públicas y el alcance y la calidad de sus prestaciones sociales se vean reducidas, especialmente en lo referido a la educación, la salud y los sistemas de seguridad social, elementos fundamentales para apoyar la plena e independiente incorporación de los jóvenes a la sociedad y facilitar el ejercicio de la ciudadanía activa.

Las consecuencias sociales y económicas derivadas del desplazamiento de las generaciones juveniles son también altas, siendo su coste más espectacular la limitación de la capacidad de la juventud para reproducir biológica y axiológicamente a la sociedad. La juventud, ese tiempo de adquisición de seguridad, identidad e independencia, necesita, por tanto, de políticas especificas que modifiquen la compleja situación en la que se encuentra y que faciliten la civilidad, es decir, la transmisión de los valores ciudadanos a las generaciones más jóvenes. 


\section{La juventud europea y el ejercicio de la ciudadanía activa}

Vinculado al tema de la emancipación juvenil, otro de los principales retos que hoy enfrentan las sociedades occidentales del siglo XXI es la formación de ciudadanos responsables capaces de participar activamente en la vida democrática de sus comunidades y en los procesos de toma de decisiones que sirvan para mejorar tanto sus condiciones de vida como las de sus conciudadanos. Los jóvenes son, en este sentido, la principal cantera para la formación de ciudadanos activos, responsables e independientes. Tal y como lo expresa Adela Cortina (1999, p. 36), "a ser ciudadano se aprende, como a casi todo lo que es importante en la vida", siendo la juventud el periodo formativo y de aprendizaje ciudadano por excelência.

Ser ciudadano es ser un miembro de pleno derecho dentro de una sociedad. Implica ser respetado y respetar. Ser ciudadano significa participar, disfrutar de unos derechos otorgados y cumplir unos deberes adquiridos. Ser ciudadano es, en definitiva, ser un miembro activo de una comunidad que fomenta la cohesión social y que promueve el sentimiento de pertenencia a esa comunidad donde tienen cabida las diferencias y las discrepancias y donde los valores democráticos son la base del desarrollo global de una sociedad cuyos miembros se caracterizan por poseer una identidad compartida (Redoli, 2002, p. 12).

Como resultado del éxito de la Revolución Americana de 1776 y de la Revolución Francesa de 1789, occidente ha dado paso a unas estructuras sociales inspiradas en los derechos que debe disfrutar todo ciudadano (Bobbio et al, 1995, p. 458-459), puesto que el ejercicio de la ciudadanía activa necesita de una fibra social que facilite la existencia de un vínculo de unión entre los ciudadanos y la comunidad democrática a la que pertenecen. Estos derechos se han ido desarrollando y consolidando a lo largo de los últimos 300 años en el mundo occidental (aunque de forma irregular) y pueden sintetizarse de la siguiente manera:

\section{1) Los derechos civiles}

Este conjunto de derechos se desarrolló durante el siglo XVIII, gracias a la consolidación de los tribunales de justicia. Los derechos civiles se caracterizan por ser aquellos derechos necesarios para obtener la libertad individual. Esta libertad, la de la persona, se basa en las libertades de expresión, de pensamiento y de reli- 
gión, y en el derecho a la propiedad y a establecer contratos, el derecho a la justicia y el derecho al trabajo en el lugar que se ha elegido. El reconocimiento de los derechos civiles significó el primer gran paso en la conformación del concepto de ciudadanía tal y como lo conocemos en la actualidad.

\section{2) Los derechos politicos}

Este conjunto de derechos se desarrolló durante el siglo XIX, gracias a la consolidación de los parlamentos y los organismos de gobierno local. Los derechos políticos aseguraron el derecho a ser elegido para ejercer el poder político y a elegir a los representantes políticos, es decir, a votar y a ser votado. La consecuencia de la emergencia de estos derechos fue el surgimiento del sufragio universal y las democracias modernas en años posteriores, debido, fundamentalmente, a que aumentó la conciencia política debido a la proliferación de asociaciones cívicas, a la fundación de nuevos partidos políticos y al nacimiento de los sindicatos obreros.

\section{3) Los derechos sociales}

Este conjunto de derechos emergió durante el siglo XX y están ligados al desarrollo de los sistemas educativos y de los servicios sociales. Se refieren, básicamente, al derecho a disfrutar de los estándares de vida que prevalecen en una sociedad dada y a participar de su herencia social. Estos derechos dotan a la ciudadanía de una igualdad de estatus, puesto que la protección que dispensan los derechos sociales está orientada a asegurar un mínimo bienestar económico para todos los ciudadanos (Marshall, 1998, p. 22-23).

El objetivo que subyace en el conjunto de los derechos sociales es que se produzca un enriquecimiento general de los ciudadanos, que se reduzcan el riesgo y la inseguridad, que se ponga límites a la disparidad de los niveles de renta entre los menos y los más afortunados y que se establezcan mecanismos estructurales de equilibrio y solidaridad entre los sanos y los enfermos, los empleados y los desempleados, los jubilados y los activos, los hombres y las mujeres, los jóvenes y los adultos, los solteros sin hijos y los padres de familia numerosa.

Los derechos sociales entroncan directamente, por tanto, con la noción de solidaridad, es decir, con la idea de que han de establecerse unos principios universales de cohesión social, ampliamente reconocidos en el Capitulo IV de la Carta de los Derechos Fundamentales de la Unión Europea, proclamada por el Consejo Europeo de Niza (Francia) en diciembre de 2000 (Oficina del Parlamento Europeo en España, 2001). Estos derechos parten de la idea de que existe una igualdad humana básica asociada a la pertenencia 
plena a una comunidad, dificultando así la conservación de desigualdades económicas y sociales (ya que deslegitiman y aumentan las probabilidades de luchar contra ellas, dando lugar de esta manera a una sociedad cohesionada). La importancia de estos derechos es especialmente relevante para los jóvenes.

A pesar de los importantes avances logrados en la consolidación de los derechos de ciudadanía, todavía se habla de ciudadanía y juventud de forma disociada, como si la condición de ciudadano tuviera una correspondencia directa con la categoría de persona adulta y la condición de joven se identificara con sujetos políticos pasivos o de segundo nivel (Storrie, 1999, p. 64).

En la Europa del siglo XXI la juventud enfrenta dificultades extraordinarias para alcanzar su independencia y su autonomía. Ante el deterioro de su situación, los jóvenes ven aceptada su condición de ciudadanos formales, es decir, de personas a las que se reconocen sus derechos y obligaciones, pero que encuentran importantes dificultades para participar activamente en la vida cívica de su sociedad debido a que las adversidades económicas y sociales limitan su independencia $\mathrm{y}$, por tanto, su autonomía personal, requisito indispensable para el ejercicio pleno del estatus de ciudadano europeo (Crouch, 1999, p. 257-285).

Un ciudadano europeo es aquel o aquella que posee la nacionalidad de cualquier Estado miembro de la Unión Europea. Ser ciudadano europeo es, en esencia, lo mismo que ser ciudadano de cualquier otra entidad política democrática del mundo, es decir, disfrutar de los derechos civiles, políticos y sociales, a la vez que se reconocen una serie de obligaciones que vinculan a las personas con su comunidad y adquieren, por tanto, un sentimiento de identidad compartida. Tal vez las peculiaridades más destacables de la ciudadanía europea sean su reciente acuñación, que los ciudadanos europeos ostentan una doble ciudadanía (la de su país y la de la Unión) y que existe lo que algunos pensadores han denominado "el modelo europeo de ciudadanía", aquel que combina un diseño de derechos conferidos desde el Estado con una ciudadanía basada en el desarrollo de la sociedad civil (Giner y Torres, 1998, p. 108). Adicionalmente, otro hecho diferencial de la ciudadanía europea es que rompe con el concepto clásico de ciudadanía, puesto que no está ligado a ningún Estado o nación en concreto, sino que se vincula con una entidad supranacional compuesta por múltiples Estados. 
La ciudadanía europea, aunque cobró entidad legal el 1 de noviembre de 1993, cuando entró en vigor el Tratado de Maastricht (también conocido como Tratado de la Unión Europea), aún se encuentra en plena fase de desarrollo. El tratado recogió en su Artículo 8 cuatro derechos esenciales de la ciudadanía europea que, si bien son muy limitados, sí instauraron definitivamente la noción de ciudadanía para todas las personas que ostenten la nacionalidad de cualquier Estado miembro de la Unión. Estos derechos son:

- el derecho de libertad de movimiento y de establecimiento dentro de todo el territorio de la UE

- el derecho a votar y a ser votado en las elecciones locales y europeas en el Estado en el que se resida

- el derecho a la protección diplomática y consular

- el derecho de petición y de recurso al defensor del pueblo europeo

No obstante, la posesión formal de la ciudadanía europea no significa necesariamente disfrutar de la ciudadanía activa, es decir, de esa ciudadanía que permite participar en los asuntos públicos de forma libre e independiente, que cualifica a los individuos a ser miembros de pleno derecho dentro de su sociedad. Los jóvenes en la actualidad necesitan de un entramado social y de un apoyo institucional que los reconozca y posibilite como ciudadanos activos. Para lograr este objetivo es necesario desarrollar una nueva cultura política participativa a escalas local, regional, estatal y europea, puesto que es la cultura política la que finalmente define la calidad y la cantidad de la participación de los ciudadanos y, por tanto, la fortaleza o la debilidad de su sociedad civil (Almond y Verba, 1992, p. 184).

Una sociedad civil ${ }^{13}$ en la que los jóvenes reconozcan su espacio permitirá favorecer la comunicación intergeneracional y robustecer los mecanismos de participación ciudadana a todos los niveles, mejorando la calidad

13 La sociedad civil es ese espacio social y político ocupado por grupos, organizaciones e individuos relativamente autónomos del Estado, que persiguen apoyar o contrarrestar determinadas acciones de éste, así como articular valores y crear nuevas asociaciones y solidaridades a través de la persecución de sus intereses. Esta definición de sociedad civil se encuentra más desarrollada en Linz, Juan J. \& Stepan, Alfred., Problems of Democratic Transition and Consolidation: Southern Europe, South America, and Post-Communist Europe, The Johns Hopkins University Press, Baltimore, 1996, p. 7. 
de la democracia y, por tanto, de las esferas pública y privada de la sociedad. Los jóvenes de "la sociedad del riesgo" necesitan, hoy más que nunca, de programas y de políticas especificas que les ayuden a ejercitar sus derechos y a cumplir con sus obligaciones como ciudadanos activos en su ciudad, en su región, en su país y, también, en las entidades supranacionales. De esta manera, los contenidos inherentes al ejercicio de la ciudadanía activa cobrarán significado dentro del conjunto de prácticas sociales que se desarrollan en la vida cotidiana de los individuos y de los grupos sociales (Morán y Benedicto, 2000, p. 161).

El movimiento asociativo juvenil europeo está dando respuestas a la problemática de la plena inclusión de los jóvenes del continente como ciudadanos activos en el seno de sus sociedades. Con el Foro Europeo de la Juventud (YFJ) al frente, el movimiento asociativo juvenil europeo lleva más de 50 años organizándose de tal manera que, hoy por hoy, se encuentra en condiciones de utilizar sus estructuras para prestar servicios formativos, para la inclusión laboral y para el ejercicio de la política activa por parte de los jóvenes asociados.

\section{Las organizaciones juveniles europeas: un nuevo impulso para el desarrollo de los servicios de juventud}

Como ya ha sido mencionado en la introducción de este trabajo, el Libro Blanco Un nuevo impulso para la juventud europea, los cinco grandes programas específicos para jóvenes de la Comisión Europea, Sócrates II, Juventud, Leonardo da Vinci II, Youth-Med y Tempus III, junto con el sistema europeo de información juvenil Eurodesk y las acciones y programas del Consejo de Europa en materia de juventud significan un importante aporte institucional en materia de elaboración de políticas públicas y de prestación de servicios de juventud (especialmente en lo referido a movilidad y formación).

No obstante, merecen mayor atención los servicios de juventud que están prestando las asociaciones juveniles europeas a sus miembros. Demasiado a menudo, los programas y los servicios oficiales prestados por instituciones públicas eclipsan la importancia y el alcance de los servicios de juventud diseñados por jóvenes e implementados por ellos mismos. Quizás 
la altísima politización de los movimientos juveniles durante la "guerra fría"14 alimentó el poso de desconfianza que recaía sobre estas estructuras pero que hoy han cambiado de faz. La caída del Muro de Berlín liberó al movimiento juvenil internacional del peso de los corsés ideológicos que aprisionaban el desarrollo autónomo de las organizaciones juveniles. La confrontación política y propagandística que llevaban a cabo la Asamblea Mundial de la Juventud, del lado occidental, y la Federación Mundial de Juventudes Democráticas, del lado comunista, dejó de tener sentido a comienzos de los años ' 90 .

Hoy en día las asociaciones juveniles en el ámbito europeo pueden fomentar un modelo participativo del sistema político de la UE y potenciar su función como estructuras mediadoras entre la población juvenil y el resto de la sociedad, dando a los jóvenes la oportunidad de acceder y hacer uso de sus cuotas efectivas de poder ciudadano (Godoy, 2000, p. 56). Adicionalmente, la juventud europea podrá forjar una identidad compartida a escala europea, una identidad que entremezcle sentimientos de pertenencia a lo local, a lo regional, a lo estatal y a lo supranacional, complementariamente.

Aunque suele decirse que la juventud es el futuro, no hay que olvidar que también es el presente. La juventud es protagonista del día a día de una Europa en construcción, cuyas formas políticas, sociales, culturales, geográficas y económicas están aún por perfilar.

Apostar por aumentar la participación activa a través del asociacionismo entre los jóvenes de la Unión Europea significa apostar por un modelo social en el que la sociedad civil ejerza plenamente sus derechos de forma organizada y profundice en el modelo de democracia participativa. La juventud, como colectivo componente de la sociedad civil, en el ejercicio de la democracia participativa puede y debe reivindicar su derecho a la participación directa en los procesos políticos, sociales y económicos que le afecten. De esta manera los jóvenes tendrán la oportunidad de desarrollar mayores niveles de intervención en su entorno y gestionar la prestación de

14 Se entiende por "guerra fría" a la tensión armamentística e ideológica entre el bloque comunista (liderado por la URSS) y el bloque capitalista (liderado por EE. UU.) que padeció el mundo entero desde el fin de la Segunda Guerra Mundial en 1945 hasta la caída del Muro de Berlín en 1989. 
servicios de juventud propios, de igual manera que los ampliamente reconocidos colegios profesionales, por poner un ejemplo, prestan sus servicios a sus colegiados, en defensa de los intereses de la profesión y promoción de la misma.

\section{El Foro Europeo de la Juventud (YFJ) y los servicios de juventud por y para el movimiento asociativo juvenil europeo}

El Foro Europeo de la Juventud (YFJ) es una plataforma juvenil internacional creada en 1996 por los consejos nacionales de juventud y las organizaciones juveniles no gubernamentales de toda Europa. Esta estructura ocupa el lugar dejado por el Consejo de los Comités Nacionales de Juventud de Europa (Council of European National Youth Committees CENYC) y el Buró Europeo de Coordinación de las Organizaciones Juveniles No-Gubernamentales (European Co-ordination Bureau of International Non-Governmental Youth Organizations - ECB), organismos que venían representando los intereses de los jóvenes desde la década de los '60. El YFJ incluye en su seno al Foro de la Juventud de la Unión Europea (YFEU), que actúa como puente entre el Foro Europeo de la Juventud y las instituciones de la Unión Europea. ${ }^{15}$

Aunque se trata de una plataforma muy interdisciplinar y heterogénea, los objetivos principales del YFJ son defender los intereses de los jóvenes europeos y promover su activa participación en el proceso de construcción de una Europa común. El YFJ trabaja con numerosas instituciones internacionales, y, principalmente, con la Unión Europea, con el Consejo de Europa y con las Naciones Unidas. Asimismo, canaliza el flujo de información y opiniones entre los jóvenes y los representantes políticos.

El YFJ cuenta en 2003 con 91 organizaciones miembro entre consejos nacionales de juventud y organizaciones juveniles no gubernamentales, quienes constituyen una estructura que representa a más de 10 millones de jóvenes de todos los países de Europa. Su fuente de financiación principal

15 Es precisamente a este órgano al que se refiere el Libro Blanco Un nuevo impulso para la juventud europea en su Apartado 7 ("Diálogo con el Foro de la Juventud de la Unión Europea”) del Anexo 2 (“Síntesis de la actividad europea en el ámbito de la juventud”). 
procede de una línea presupuestaria directa asignada por la Comisión Europea que también es aprobada por el Consejo de la Unión Europea y por el Parlamento Europeo. Asimismo, el YFJ recibe aportaciones económicas periódicas procedentes del Consejo de Europa. ${ }^{16}$ Idiomáticamente, el inglés y el francés son las dos principales lenguas utilizadas tanto interna como externamente por el Foro Europeo de la Juventud.

El Foro Europeo de la Juventud es dirigido políticamente por un Buró compuesto por jóvenes representantes de organizaciones miembro que representan la diversidad y la pluralidad del movimiento asociativo juvenil. El buró está compuesto por un presidente, dos vice-presidentes y ocho miembros que, por un período de dos años, asumen la responsabilidad de diseñar y ejecutar los planes de trabajo y las decisiones adoptadas tanto en la Asamblea General como en el Consejo de Miembros del YFJ. En el plano técnico, un Secretario General y un número variable de técnicos (desde traductores hasta contables) son los responsables de trasvasar a la acción el plan de trabajo y las decisiones políticas tomadas por el Buró del YFJ.

Adicionalmente, el Foro Europeo de la Juventud cuenta con cuatro Unidades de Trabajo y cuatro Cuerpos Estatutarios que complementan el trabajo desarrollado por el Buró. Las cuatro Unidades de Trabajo son las siguientes: a) Grupo de Trabajo sobre la Educación, b) Grupo de Formadores, c) Grupo sobre el Desarrollo del Trabajo Juvenil, y d) Comité de Cooperación y Coordinación Global. Los cuatro Cuerpos Estatutarios son los siguientes: a) Consejo de la Comisión de Asuntos Europeos (COECOM), b) Comisión de Asuntos de la Unión Europea (EUACOM), c) Comisión de Control Financiero (FCC), y d) Cuerpo Consultivo sobre Solicitudes de Admisión (CBMA).

Conscientes de la multiplicidad de problemas que enfrentan los jóvenes asociados europeos, el Foro Europeo de la Juventud diseña planes de trabajo que, además de promover líneas de actuación política, persiguen capacitar a la juventud europea para el ejercicio activo de la ciudadanía. De esta manera,

16 Con respecto a los ingresos y los gastos del Foro Europeo de la Juventud, cabe decir que son exhaustivamente supervisados por un equipo interno de auditores elegido por las organizaciones miembro del YFJ. Asimismo, anualmente se contratan los servicios de una compañía auditora externa y se facilita también la auditoría a los servicios financieros de la Comisión Europea. 
los principales ejes de acción del YFJ para los próximos años son mejorar las condiciones de vida de los jóvenes europeos, facilitar su inserción laboral (agilizando la emancipación juvenil y, por tanto, la autonomía de los jóvenes), robustecer las políticas y los servicios de juventud europeos (especialmente en materia de educación formal, no formal, movilidad y financiación de organizaciones juveniles) y aumentar la cooperación internacional. ${ }^{17}$

Como no podía ser de otra manera, entre la diversidad de actividades, planes y programas del YFJ, se encuentran la prestación de servicios que cubren un amplio abanico de demandas. Los principales servicios prestados por el Foro Europeo de la Juventud son los siguientes:

\section{a) Servicios editoriales}

El YFJ edita, principalmente, dos publicaciones con carácter regular: la revista bimensual "Youth Opinion", publicada en inglés y francés, y el boletín informativo "FLASH", referido a las actividades desarrolladas bajo cada presidencia rotativa del Consejo de la Unión Europea. Asimismo, el YFJ gestiona una web propia <www.youthforum.org> desde la que pueden descargarse todos los documentos de trabajo de la organización.

\section{b) Servicios orientados hacia la promoción de los Derechos Humanos y la igualdad}

El YFJ supervisa las políticas implementadas por las principales instituciones internacionales que trabajan sobre la defensa de los Derechos Humanos, persiguiendo la eliminación del racismo y la xenofobia, así como de la discriminación de las mujeres jóvenes. Asimismo, diseña y ejecuta políticas de defensa de los Derechos Humanos para el colectivo de jóvenes y de sus organizaciones y participa activamente en el Programa de Educación para los Derechos Humanos del Consejo de Europa y en las Cumbres organizadas por las Naciones Unidas. Adicionalmente, organiza seminarios, cursos, conferencias y ciclos formativos referidos a la defensa de los Derechos Humanos y la igualdad entre la juventud.

17 Tal y como se reconoce en el Plan de Trabajo 2003-2004, adoptado en Asamblea General en Malta entre el 14 y el 16 de noviembre de 2002. Este Plan de Trabajo puede descargarse en la dirección $<$ www.youthforum.org $>$. 
c) Servicios orientados hacia la promoción de la ciudadanía activa y el aprendizaje continuo

El YFJ promueve la educación no-formal ${ }^{18}$ como forma de aprendizaje que capacita a los jóvenes para ejercitar responsable y participativamente sus derechos y deberes ciudadanos. En este sentido, el YFJ promueve numerosas campañas de lobby en el ámbito europeo para procurar mayores niveles de reconocimiento de la educación no formal por parte de empresas e instituciones públicas. Esta perspectiva responde a la aproximación teórica hacia la educación como un proceso continuo que comienza en la cuna y no termina hasta llegado el final de la vida.

\section{d) Servicios de lobby}

El YFJ defiende los intereses del colectivo de jóvenes asociados en Europa a todos los niveles, prestando especial atención a las demandas de los consejos nacionales de juventud y de las organizaciones juveniles no gubernamentales miembro. En este sentido, el Foro Europeo de la Juventud mantiene estrechos y constantes contactos con diferentes agencias del Sistema de Naciones Unidas, con el Consejo de Europa y con las instituciones de la Unión Europea (especialmente con la Comisión, con el Parlamento y con el Consejo). Asimismo, elabora documentos propositivos públicos que son remitidos a todas aquellas instituciones involucradas en la toma de decisiones sobre políticas públicas de juventud.

\section{e) Servicios para la coordinación de la cooperación juvenil a nivel global}

El YFJ ha desarrollado un área de cooperación juvenil a nivel global con la finalidad de vincular las organizaciones juveniles europeas a todas aquellas instituciones y contrapartes que, en cualquier continente, sean susceptibles de promover positivamente al colectivo de jóvenes. Asimismo, esta área de cooperación persigue establecer redes estables entre las organizaciones juveniles del continente europeo con otras de otras latitudes. En un mundo donde el fenómeno de la globalización cobra cada vez más importancia, el área de cooperación juvenil a nivel global se ha convertido en

18 La educación no formal es el aprendizaje que se da fuera de las instituciones educativas formales, en estructuras tales como la familia o las organizaciones juveniles no gubernamentales. 
una de las piedras angulares del YFJ. Un ejemplo de esta línea de servicios prestados al movimiento asociativo juvenil se reflejó en la Cumbre Mundial sobre Desarrollo Sostenible celebrada en Johanesburgo (Sudáfrica) entre el 26 de agosto y el 4 de septiembre de 2002, donde el YFJ desempeñó un activo papel haciendo visibles las reivindicaciones del movimiento asociativo juvenil a escala planetaria.

\section{f) Servicios de asistencia técnica a las entidades miembro}

El YFJ incluye entre uno de sus principales activos la prestación de servicios tanto a consejos nacionales de juventud como a organizaciones juveniles no gubernamentales. El departamento encargado de centralizar estos trabajos se encarga, fundamentalmente de tres áreas: (a) el suministro de servicios de formación, (b) el suministro de asistencia técnicolegislativa, y, (c) el suministro de asistencia en materia de cooperación local y regional en toda Europa (aunque prestando especial atención a las regiones de Europa Central, de Europa del Este y de Europa del Sudeste). Fruto del activo funcionamiento de estos servicios ha sido el Balkan Youth Project.

\section{g) Servicios de empleo y asuntos sociales}

El YFJ considera al empleo como una de las principales preocupaciones de los jóvenes del continente. Dados los altos niveles de desempleo juvenil (tal y como ha sido expuesto a lo largo de este trabajo) que afectan sobremanera al proceso de emancipación de las cohortes más jóvenes, el YFJ organiza conferencias, cursos y seminarios sobre la importancia del proceso transicional desde el sistema educativo hasta el mercado de trabajo. Es precisamente en ese momento cuando el joven se encuentra más desprotegido y vulnerable a la exclusión social, a sufrir explotación laboral, a percibir bajos salarios o a sufrir empleos altamente inestables. Reconociendo el deterioro de las condiciones laborales para el colectivo de jóvenes europeo durante los últimos 15 años, el YFJ promueve el desarrollo de políticas públicas que incrementen los niveles de protección social para las cohortes de menor edad que entran en el mercado laboral. Un resultado del ejercicio de estos servicios fue la Conferencia sobre la Situación Social de los Jóvenes en una Europa Ampliada, organizada en septiembre de 2001 por el YFJ. 


\section{Conclusiones}

El conjunto de la serie de servicios de juventud prestados por el Foro Europeo de la Juventud está consiguiendo lo que muchas instituciones públicas parecen no resolver: capacitar a los jóvenes para participar, para ser plenos ciudadanos activos al servicio de su comunidad y, especialmente, para dotarles de recursos que faciliten su plena inserción laboral $\mathrm{y}$, por tanto, su autonomía personal. Los servicios de juventud prestados por el Foro Europeo de la Juventud llevan años demostrando que los dos grandes problemas expuestos a lo largo de este trabajo, la dilatación del proceso de obtención de la autonomía y los obstáculos que encuentran los jóvenes para el ejercicio de la ciudadanía activa en la Unión Europea, pueden resolverse dando mayores cuotas de poder, de responsabilidad y de libertad a los miembros del movimiento asociativo juvenil.

Tanto los consejos regionales y nacionales de juventud como las organizaciones juveniles no gubernamentales son incuestionables agentes de desarrollo. Facilitar la prestación de servicios de juventud a estas estructuras redundará, indefectiblemente, en ventajas para sus más directos destinatarios, los jóvenes, quienes han demostrado que pueden ser responsables del diseño y de la ejecución de servicios que den respuestas a sus propios problemas.

No basta con desarrollar servicios de juventud desde las instituciones públicas o las organizaciones no gubernamentales. Es necesario facilitar que estos servicios sean también diseñados y ejecutados por aquellos colectivos de ciudadanos destinatarios de sus beneficios (jóvenes, ancianos, mujeres, inmigrantes, desempleados o cualquier otro colectivo con capacidad para organizarse). De esta manera se estará robusteciendo la democracia participativa que, como es sabido, se centra en la acción directa de los ciudadanos en el proceso de toma de decisiones. Bajo este enfoque, la democracia tiene que concebirse como la creación de oportunidades (Sen, 2000, p. 193), oportunidades que servirán para potenciar el principio de igualdad que fomentará la cohesión social y la inclusión. Tal y como lo define el sociólogo Anthony Giddens: 
Inclusión se refiere en su sentido más amplio a la ciudadanía, a los derechos y deberes civiles y políticos que todos los miembros de una sociedad deberían tener, no sólo formalmente, sino como una realidad de sus vidas. También se refiere a las oportunidades y a la integración en el espacio público. En una sociedad en la que el trabajo sigue siendo esencial para la autoestima y el nivel de vida, el acceso al trabajo es un ámbito principal de oportunidades. La educación es otro, y lo sería incluso aunque no fuera tan importante para las posibilidades de empleo, para las que es determinante (Giddens, 1999, p. 123).

Los servicios de juventud prestados por la propias asociaciones juveniles fomentan la participación y el aprendizaje y, por tanto, la inclusión social y, consecuentemente, facilitan los procesos de participación. La participación, entendida como proceso, es inherente a cualquier ciudadano o grupo de ciudadanos, más allá de su edad, procedencia, género o preferencia sexual. El movimiento asociativo juvenil puede (y debe) prestar servicios a los propios jóvenes principalmente porque es parte de la sociedad civil, una parte muy importante por su capacidad de generar propuestas, contenidos y capacitar capital humano caracterizado por jóvenes habituados a trabajar en equipo, con iniciativa, acostumbrados a organizar y a desenvolverse en el marco de instituciones y organizaciones, con visión de los procesos globales y con experiencia en manejar recursos, tomar decisiones y resolver conflictos.

Si la Unión Europea del siglo XXI quiere apostar por dar un verdadero impulso a la juventud europea, debe empezar por impulsar el desarrollo de los servicios de juventud prestados por el propio movimiento asociativo juvenil europeo.

\section{Referencias}

ABERCROMBIE, N. HILL, S. y TURNER, B. S. Diccionario de Sociología. Madrid: Editorial Cátedra, 1992.

ALCÁNTARA SÁEZ, M. (ed.). Sistemas politicos de la Unión Europea. Tirant lo Blanch: Valencia, 2000.

ALMOND, G. A. y VERBA, S. La cultura política. En: BATLle, A. (ed.). Diez textos básicos de ciencia política. Barcelona: Editorial Ariel, 1992.

ALONSO, L. E. El marco social del empleo juvenil: Hacia una reconstrucción del lugar del trabajo en las sociedades complejas. En: CACHÓN, L. (dir.). Juventudes y empleos: perspectivas comparadas. Madrid: Instituto de la Juventud, 2000.

BAUMAN, Z. La sociedad individualizada. Madrid: Cátedra, 2001. 
BECK, U. La sociedad del riesgo: hacia una nueva modernidad. Barcelona: Paidós, 1998.

Un nuevo mundo feliz: La precariedad del trabajo en la era de la globalización. Barcelona: Editorial Paidós, 2000.

BELTRÁN, E. y SEMPERE, D. Las organizaciones del Consejo de la Juventud de España. Un análisis humano y organizativo. Madrid: Consejo de la Juventud de España, 2002.

BOBBIO, N., MATTEUCCI, N. y PASQUINO, G. (eds.). Diccionario de Política. México: Siglo Veintiuno Editores, 1995.

BORJA, J., Geneviève D., y Peugeot V. La ciudadanía europea. Barcelona: Ediciones Península, 2001.

BRU, C. M. La ciudadanía europea. Madrid: Editorial Sistema, 1994.

CACHÓN, L. Las políticas de transición: entre las biografías individuales y los mercados de trabajo. Estrategias de los actores, lógicas y políticas de empleo juvenil en Europa. Documento de trabajo presentado en la Conferencia europea para investigadores y técnicos "Jóvenes y políticas de transición en Europa". Madrid, 6-8 de junio de 2002.

CARNOY. M. El trabajo flexible en la era de la información. Madrid: Alianza, 2001.

CASTELLS, M. La era de la información: economía, sociedad y cultura. Madrid: Alianza, 2000.

CENTRO DE INVESTIGACIONES SOCIOLÓGICAS (CIS), Expectativas y preocupaciones de los jóvenes, estudio n⿳ํㅡㄹ 2221, Madrid, 1996.

COLECTIVO IOE (Actis, Walter, Pereda, Carlos y De Prada, Miguel A.), Condiciones de trabajo de los jóvenes, edita el Consejo de la Juventud de España, Madrid, 1987.

COMISIÓN EUROPEA (Dirección General de Educación y Cultura), Libro Blanco de la Comisión Europea: Un nuevo impulso para la juventud europea. Luxemburgo: Oficina de Publicaciones Oficiales de las Comunidades Europeas, 2002.

CONSEJO DE LA JUVENTUD DE ESPAÑA, Políticas integrales de juventud en el ámbito rural, CJE, Madrid, 2002.

CORTINA, A. Ciudadanos del mundo: hacia una teoría de la ciudadanía. Madrid: Alianza Editorial, 1999.

_. Los ciudadanos como protagonistas. Barcelona: Galaxia Gutemberg, 1999.

CROUCH, C. La ampliación de la ciudadanía social y económica y la participación. En: GARCÍA, S. y LUKES, S. (eds.). Ciudadania: justicia social, identidad y participación. Madrid: Editorial Siglo Veintiuno, 1999. 
DAHL, R. A. Democracia: una guía para los ciudadanos. Madrid: Editorial Taurus, 1999.

DIRECCIÓN GENERAL DE COOPERACIÓN CON EL ESTADO Y ASUNTOS EUROPEOS, Guía práctica sobre las politicas de la Unión Europea, Comunidad de Madrid, Madrid, 2000.

EQUIPO DE INVESTIGACIÓN DE PROVIVIENDA, Vivienda y juventud en el año 2000, Instituto de la Juventud, Madrid, 2001.

EUROPEAN COMMISSION DIRECTORATE-GENERAL FOR EDUCATION AND CULTURE, Freeze-frame on Europe's Youth... for a new impetus: The main results of the Eurobarometer 2001 survey on Youth, Office for Official Publications of the European Communities, Luxembourg, 2002.

EUROSTAT \& EUROPEAN COMMISSION DIRECTORATE GENERAL FOR EMPLOYMENT AND SOCIAL AFFAIRS, The Social Situation in the European Unión 2001, Office for Official Publications of the European Communities, Luxembourg, 2002.

FUNES, J. Migración y adolescencia. En: VV.AA. La inmigración extranjera en España: los retos educativos. Barcelona: Fundación la Caixa, 1999.

GARRIDO, L. y REQUENA, M. La emancipación de los jóvenes en España. Madrid: Ministerio de Trabajo y Asuntos Sociales/Instituto de la Juventud, 1996.

GELLNER, E. Condiciones de la libertad: La sociedad civil y sus rivales. Barcelona: Editorial Paidós, 1996.

GIDDENS, A. La tercera vía: La renovación de la socialdemocracia. Madrid: Taurus, 1999.

. Modernidad e identidad del yo: El yo y la sociedad en la época contemporánea, Barcelona: Editorial Península, 1996.

. Sociología. Madrid: Alianza Editorial Textos, 1993.

GIL CALVO, E. Identidades complejas y cambio biográfico. En: VV. AA. Estructura y cambio social. Madrid: Centro de Investigaciones Sociológicas, 2001.

GINER, S. Lamo de Espinosa, E. y Torres, C. (eds.). Diccionario de Sociología. Madrid: Alianza Editorial, 1998.

GODOY, A. y FRANCO, P. Cultura participativa y asociacionismo juvenil. Madrid: Consejo de la Juventud de España, 2000.

HERNÁNDEZ ARISTU, J. Jóvenes entre la familia, la formación y el empleo Estructuras de apoyo a sus transiciones. En: VV.AA. Jóvenes y transiciones a la vida adulta en Europa, Revista de Estudios de Juventud, $\mathrm{n}^{\mathrm{o}}$ 56, Instituto de la Juventud, Madrid, 2002. 
HOOGHE, L. y MARKS, G. Una politeya en formación: pugnas sobre la integración europea. En: Llamazares, I. y Reinares, F. (eds.). Aspectos políticos y sociales de la integración europea. Valencia: Editorial Tirant lo Blanch, 1999.

IARD, Estudio sobre la situación de los jóvenes y la política de juventud en Europa, Milán, 2001.

INGLEHART, R. El cambio cultural en las sociedades industriales avanzadas. Valencia: Ed. Siglo XXI, 1991.

IRIS, Misleading Trayectories?: An Evaluation of the Unintended Effects of Labour Market Integration Policies for Young Adults in Europe. Documento de trabajo presentado en la Conferencia europea para investigadores y técnicos "Jóvenes y politicas de transición en Europa". Madrid, 6-8 de junio de 2002.

LARSÉN, M. Speak Out! on European Citizenship. London: Institute for Citizenship, 2000.

LEHNING, P. B. Towards a Multi-cultural Civil Society: The Role of Social Capital and Democratic Citizenship. En: BERNARD, A., HELMICH, H. y Lehning, P. Civil Society and International Development. Paris: North-South Centre of the Council of Europe \& Development Centre of the Organization for Economic Co-operation and Development, 1998.

LINZ, J. J. y STEPAN, A. Problems of Democratic Transition and Consolidation: Southern Europe, South America, and Post-Communist Europe. Baltimore: The Johns Hopkins University Press, 1996.

LLAMAZARES, I. y REINARES, F. (eds.). Aspectos políticos y sociales de la integración europea. Valencia: Editorial Tirant lo Blanch, 1999.

MACHADO PAIS, J. Laberintos de vida: paro juvenil y rutas de salida (jóvenes portugueses). En: VV. AA. Jóvenes y transiciones a la vida adulta en Europa. Revista de Estudios de Juventud, nํํ56, Instituto de la Juventud, Madrid, 2002.

MARÍ-KLOSE, M. y NOS, A. Itinerarios vitales: educación, trabajo y fecundidad de las mujeres. Madrid: Centro de Investigaciones Sociológicas, 1999.

MARSHALL, T. H. Ciudadanía y clase social. Madrid: Alianza Editorial, 1998.

MARTÍN SERRANO, M. y VELARDE HERMIDA, O. Informe juventud en España 2000. Madrid: Instituto de la Juventud, 2001.

MARTÍN SERRANO, M. La prolongación de la etapa juvenil de la vida y sus efectos en la socialización. En: VV.AA. Jóvenes y transiciones a la vida adulta en Europa. Revista de Estudios de Juventud, ํㅜ56, Instituto de la Juventud, Madrid, 2002.

- L Las transiciones juveniles y las contradicciones de la socialización. Documento de trabajo presentado en la Conferencia europea para investigadores y técnicos "Jóvenes y políticas de transición en Europa". Madrid, 6-8 de junio de 2002. 
MARTÍNEZ, A. y MÉNDEZ, M. Las elecciones al Parlamento Europeo 1999. Valencia: Editorial Tirant lo Blanch, 2000.

MATEOS, A. y MORAL, F. El cambio en las actitudes y los valores de los jóvenes. Madrid: Instituto de la Juventud, 2002

MCNEISH, Wallace \& L'ONCLE, Patricia. State Policy and Youth Unemployment in the European Union: Integration Through Training? Comparing the Effectiveness of Strategies to Promote the Integration of Unemployed Young People in the Aftermath of the 1997 Luxembourg Summit". Documento de trabajo presentado en la Conferencia europea para investigadores y técnicos "Jóvenes y políticas de transición en Europa". Madrid, 6-8 de junio de 2002.

MEIL, G. Imágenes de la solidaridad familiar. Madrid: Centro de Investigaciones Sociológicas, 1995.

MENCÍA, E. Educación cívica del ciudadano europeo: conocimiento de Europa y actitudes europeistas en el currículo. Madrid: Editorial Narcea, 1996.

MORAL, F. Los desempleados en la unidad familiar: proveedores y dependientes. Revista española de investigaciones sociológicas, ํㅡ 86, Madrid, 1999.

MORÁN, M. L. y BENEDICTO, J. Jóvenes y ciudadanos. Madrid: Instituto de la Juventud, 2000.

MORATA, F. La Unión Europea: procesos, actores y políticas. Barcelona: Editorial Ariel, 1998.

MORCH, M., MORCH, S., BOHNISCH, L., SEIFERT, H., Lopez, A., BASCUÑAN, J. y GIL, G. Sistemas educativos en sociedades segmentadas: 'trayectorias fallidas' en Dinamarca, Alemania Oriental y España. En: VV. AA. Jóvenes y transiciones a la

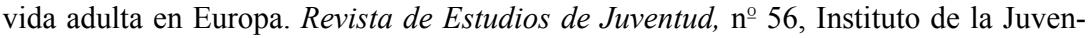
tud, Madrid, 2002.

MOYANO, E. El concepto de capital social y su utilidad para el análisis de las dinámicas del desarrollo. Revista de Fomento Social, nํ 56, 2000.

OFICINA DE PUBLICACIONES OFICIALES DE LAS COMUNIDADES EUROPEAS, Un futuro sin fronteras: Europa para los jóvenes, Luxemburgo, 1999.

OFICINA DEL PARLAMENTO EUROPEO EN ESPAÑA, Guía didáctica: propuestas de trabajo educativo para conocer la Carta de los Derechos Fundamentales de la Unión Europea, Madrid, 2001.

OLDFIELD, A. Citizenship and Community. London: Routledge, 1990.

OSLER, A. The Contribution of Community Action Programmes in the Fields of Education, Training, and Youth to the Development of Citizenship with a European Dimension. Birmingham: University of Birmingham, 1997.

PANIAGUA SOTO, J. L. Informe sobre politicas de juventud en España. Madrid: Instituto de la Juventud, 1999. 
PASQUINO, G. y otros. Manual de ciencia política. Madrid: Alianza Universidad Textos, 1995.

PRATS, J. Los jóvenes ante el reto europeo. Barcelona: Fundación la Caixa, 2001.

PROCACCI, G. Ciudadanos pobres, la ciudadanía social y la crisis de los estados del bienestar. En: GARCÍA, S. y LUKES, S. (comps.) Ciudadanía: justicia social, identidad y participación. Madrid: Siglo Veintiuno de España Editores, 1999.

REDOLI MORCHÓN, D. Juventud ciudadana en la Unión Europea: Guía didáctica para una ciudadanía europea. Madrid: Consejo de la Juventud de España, 2002.

ROCHE, J. y TUCKER, S. (eds.). Youth in Society. The Open University Press, 1999.

ROMÁN, P. y Ferri, J. (eds.). Los movimientos sociales: Conciencia y acción de una sociedad politizada. Madrid: Consejo de la Juventud de España, 2002.

RUIZ, J., Quintás, S. y Sánchez, Y. El presente de los jóvenes. En: Investigación y Marketing, $\mathrm{n}^{\circ}$ 52, septiembre, 1996.

SANAHUJA, J. A. Altruismo, mercado y poder: El Banco Mundial y la lucha contra la pobreza. Barcelona: Intermón-Oxfam, 2001.

SÁNCHEZ-CUENCA, I. El déficit democrático de la Unión Europea. En: LLAMAZARES, I. y REINARES, F. Aspectos politicos y sociales de la integración europea. Valencia: Editorial Tirant lo Blanch, 2000.

SANZ, J. y VILADEMUNT, L. Los jóvenes parlamentarios en España. Barcelona: Fundación Francesc Ferrer i Guardia, 2001.

SEN, A. Desarrollo y libertad. Barcelona: Planeta, 2000.

SENNET, R. La corrosión del carácter: Las consecuencias personales del trabajo en el nuevo capitalismo. Barcelona: Editorial Anagrama, 2000.

SERRANO PASCUAL, A. Procesos paradójicos de construcción de la juventud en un contexto de crisis del mercado de trabajo. REIS, ํำ 71- 72, 1995.

SERRANO, J. y SEMPERE, D. La participación juvenil en España. Barcelona: Fundació Ferrer y Guardia, 1999.

SURIAN, A. Education for Global Citizenship: Examples of Good Practice in Global Education in Europe (Education and Training for Global Interdependence). Lisbon: North-South Centre of the Council of Europe, 1997.

TRILLA, C. La politica de vivienda en perspectiva europea comparada. Barcelona: Fundación La Caixa, 2001.

VV. A.A. Informe España 2002. Una interpretación de su realidad social. Madrid: Fundación Encuentro, 2002.

WIHTOL DE WENDEN, C. La ciudadanía europea. Barcelona: Edicions Bellaterra, 1999. 